\title{
Independent Subspace Analysis on Innovations
}

\author{
Barnabás Póczos, Bálint Takács, and András Lórincz* \\ Eötvös Loránd University, Pázmány P. sétány 1/C, \\ Budapest, Hungary 1117 \\ barn@ludens.elte.hu, \\ \{takbal, andras.lorincz\}@elte.hu, \\ http://nipg.inf.elte.hu/
}

\begin{abstract}
Independent subspace analysis (ISA) that deals with multidimensional independent sources, is a generalization of independent component analysis (ICA). However, all known ISA algorithms may become ineffective when the sources possess temporal structure. The innovation process instead of the original mixtures has been proposed to solve ICA problems with temporal dependencies. Here we show that this strategy can be applied to ISA as well. We demonstrate the idea on a mixture of $3 \mathrm{D}$ processes and also on a mixture of facial pictures used as twodimensional deterministic sources. ISA on innovations was able to find the original subspaces, while plain ISA was not.
\end{abstract}

\section{Introduction}

Independent Component Analysis (ICA) 112 aims to recover linearly or nonlinearly mixed independent and hidden sources. There is a broad range of applications for ICA, such as blind source separation and blind source deconvolution [3], feature extraction [4], denoising [5]. Particular applications include, e.g., the analysis of financial data [6, data from neurobiology, fMRI, EEG, and MEG (see, e.g., [7/8] and references therein). For a recent review on ICA see [9].

Original ICA algorithms are 1-dimensional: all sources are assumed to be independent real valued stochastic variables. However, applications where not all, but only certain groups of the sources are independent may have high relevance in practice. In this case, independent sources can be multi-dimensional. Consider, e.g., the generalization of the cocktail-party problem, where independent groups of musicians are playing at the party. This is the subject of Independent Subspace Analysis (ISA), an extension of ICA, also called Multi-dimensional Independent Component Analysis 10[11. Efforts have been made to develop ISA algorithms 10111213 14 15 16]. Certain approaches use 2-dimensional Edgeworth expansion [12 leading to sophisticated equations. They have not been extended to 3 or higher dimensions. Another suggestion is to start with ICA and then permute the columns of the mixing matrix to find the best ISA estimation [10. This case has not been worked out and permutations may not be general

* Corresponding author.

J. Gama et al. (Eds.): ECML 2005, LNAI 3720, pp. 698706 2005.

(C) Springer-Verlag Berlin Heidelberg 2005 
enough. Another recent approach searches for independent subspaces via kernel methods [14].

These ISA algorithms all have a serious drawback: they require independently and identically distributed (i.i.d.) sources. For the ICA problem several authors 17/1819 suggested that the innovation process, instead of the original mixtures, could be used if input had a temporal structure. The innovation process can be calculated relatively easily, if one assumes that the sources are AR processes. Here we propose to compute the innovation process of the mixed signal by assuming an underlying autoregressive (AR) process first, and then to apply our new, efficient ISA algorithms 1516].

The paper is built as follows: Section 2 is an overview of the ISA problem. Section 3 presents the motivation for using the innovation process. This section describes the corresponding ISA algorithm. Numerical simulations are presented in Section 4. Short discussion and conclusions are provided in Section 5

\section{The ISA Model}

Assume we have $d$ of $m$-dimensional independent sources denoted by $\boldsymbol{y}^{1}, \ldots, \boldsymbol{y}^{d}$, respectively, where $\boldsymbol{y}^{i} \in \mathbb{R}^{m}$. Let $\boldsymbol{y}=\left[\left(\boldsymbol{y}^{1}\right)^{T}, \ldots,\left(\boldsymbol{y}^{d}\right)^{T}\right]^{T} \in \mathbb{R}^{d m}$, where superscript $T$ stands for transposition. We assume that these sources are hidden and we can only observe the following signal

$$
\boldsymbol{x}=\boldsymbol{A y}
$$

where $\boldsymbol{A} \in \mathbb{R}^{d m \times d m}$. The task is to recover hidden source $\boldsymbol{y}$ and mixing matrix $\boldsymbol{A}$ given the observed signal $\boldsymbol{x} \in \mathbb{R}^{d m}$. In the ISA model we assume that $\boldsymbol{y}^{i} \in \mathbb{R}^{m}$ is independent of $\boldsymbol{y}^{j} \in \mathbb{R}^{m}$ for $i \neq j$. For the special case of $\mathrm{m}=1$, the ICA problem is recovered.

In the ICA problem, given the signals, sources $y^{i}(i=1, \ldots, d)$ can be recovered only up to sign, up to arbitrary scaling factors, and up to an arbitrary permutation. The ISA task has more freedom; signals $\boldsymbol{y}^{i}$ can be recovered up to an arbitrary permutation and an $m$-dimensional linear, invertible transformation. It is easy to see this by considering matrix $\boldsymbol{C} \in \mathbb{R}^{d m \times d m}$ made of a permutation matrix of size $d \times d$, where each element is made of an $m \times m$ block-matrix having invertible $\boldsymbol{C}_{i}$ blocks replacing the non-zero elements of the permutation matrix. Then, $\boldsymbol{x}=\boldsymbol{A} \boldsymbol{y}=\boldsymbol{A} \boldsymbol{C}^{-1} \boldsymbol{C} \boldsymbol{y}$, and because $\boldsymbol{y}^{i}$ is independent of $\boldsymbol{y}^{j}$, thus $\boldsymbol{C}_{i} \boldsymbol{y}^{i}$ is independent of $\boldsymbol{C}_{j} \boldsymbol{y}^{j} \forall i \neq j$. That is, in the ISA model, matrices $\boldsymbol{A}$ and $\boldsymbol{A} \boldsymbol{C}^{-1}$ and sources $\boldsymbol{y}^{i}$ and $\boldsymbol{C}_{i} \boldsymbol{y}^{i}$ are indistinguishable. This ambiguity of the ISA task can be lowered by assuming $E\{\boldsymbol{y}\}=\mathbf{0}$, and $E\left\{\boldsymbol{y} \boldsymbol{y}^{T}\right\}=\boldsymbol{I}_{m d}$, where $E$ is the expected value operator, $\boldsymbol{I}_{n}$ is the $n$-dimensional identity matrix. Similarly, by scaling observed signal $\boldsymbol{x}$, one can assure that $E\{\boldsymbol{x}\}=\mathbf{0}$, and $E\left\{\boldsymbol{x} \boldsymbol{x}^{T}\right\}=\boldsymbol{I}_{m d}$, which is called the whitening of the inputs. Then, Eq. (1) ensures that $E\left\{\boldsymbol{x} \boldsymbol{x}^{T}\right\}=$ $\boldsymbol{A} E\left\{\boldsymbol{y} \boldsymbol{y}^{T}\right\} \boldsymbol{A}^{T}$ and $\boldsymbol{I}_{m d}=\boldsymbol{A} \boldsymbol{A}^{T}$. It then follows that under our assumptions, signals $\boldsymbol{y}^{i}$ can be recovered up to permutation and up to $m$-dimensional orthogonal transformation in the ISA problem. In other words, if $\boldsymbol{C}_{i} \in \mathbb{R}^{m \times m}$ 
is an arbitrary orthogonal matrix, then signals $\boldsymbol{x}$ will not provide information whether the original sources correspond to $\boldsymbol{y}^{i}$ or, instead, to $\boldsymbol{C}_{i} \boldsymbol{y}^{i}$. For the $1 \mathrm{D}$ case this is equivalent to the uncertainty that $\boldsymbol{C}_{i}=1$ or $\boldsymbol{C}_{i}=-1$. That is, in $1 \mathrm{D}$, the sign of $y^{i}$ is not determined. Thus, without any loss of generality, it is satisfactory to restrict the search for mixing matrix $\boldsymbol{A}$ (or, for its inverse, i.e., for separation matrix $\boldsymbol{W}$ ) to the set of orthogonal matrices.

\subsection{The ISA Objective}

We introduce the ISA objective subject to the constraint of $\boldsymbol{W}^{T} \boldsymbol{W}=\boldsymbol{I}_{m d}$. The separation matrix $\boldsymbol{W}$ is amongst the global minima of this ISA objective function. Let $I\left(\boldsymbol{y}^{1}, \ldots, \boldsymbol{y}^{d}\right)$ denote the mutual information between vectors $\boldsymbol{y}^{1}, \ldots, \boldsymbol{y}^{d} \in \mathbb{R}^{m}$. Further, let $H(\boldsymbol{y})$ denote the joint Shannon-entropy of vectorvalued stochastic variable $\boldsymbol{y}$. Let $\boldsymbol{y}=\boldsymbol{W} \boldsymbol{x}$. Then

$$
I\left(\boldsymbol{y}^{1}, \boldsymbol{y}^{2}, \ldots, \boldsymbol{y}^{d}\right)=-H(\boldsymbol{x})+\log |\boldsymbol{W}|+H\left(\boldsymbol{y}^{1}\right)+\ldots+H\left(\boldsymbol{y}^{d}\right)
$$

Our task is to minimize (2). However, $H(\boldsymbol{x})$ is constant and $\boldsymbol{W}^{T} \boldsymbol{W}=\boldsymbol{I}$. Thus $\log |\boldsymbol{W}|=0$ and the minimization of (2) is equivalent to the minimization of

$$
J(\boldsymbol{W}) \doteq H\left(\boldsymbol{y}^{1}\right)+\ldots+H\left(\boldsymbol{y}^{d}\right) .
$$

\subsection{Multi-dimensional Entropy Estimation}

Recently, we have developed efficient solutions to the ISA problem [15]16], which are based on efficient multi-dimensional estimations of the entropy $H\left(\boldsymbol{y}^{i}\right)$. Under mild assumptions, the Beadword-Halton-Hammersley theorem 2021] approximates Rényi's $\alpha$-entropy, which in turn can be used for approximation of the Shannon entropy. This estimation is asymptotically unbiased and strongly consistent [20]. We modify the result of this theorem by a monotone increasing transformation and propose the following estimation [15]: Let $\left\{\boldsymbol{y}^{i}(1), \ldots, \boldsymbol{y}^{i}(n)\right\}$ be an i.i.d. sample set from distribution $\boldsymbol{y}^{i}$. Let $\mathcal{N}_{k, j}^{i}$ be the $k$ nearest neighbors of $\boldsymbol{y}^{i}(j)$ in this sample set. Then a possible estimation of $H\left(\boldsymbol{y}^{i}\right)$ up to an irrelevant additive and multiplicative constant is the following:

$$
\hat{H}^{1}\left(\boldsymbol{y}^{i}\right) \doteq \lim _{\gamma \rightarrow 0} \sum_{j=1}^{n} \sum_{\boldsymbol{z} \in \mathcal{N}_{k, j}^{i}}\left\|\boldsymbol{z}-\boldsymbol{y}^{i}(j)\right\|^{\gamma}
$$

In [15] we also derived another estimation for the entropy:

$$
\hat{H}^{2}\left(\boldsymbol{y}^{i}\right) \doteq \sum_{j=1}^{n} \sum_{\boldsymbol{z} \in \mathcal{N}_{k, j}^{i}} \log \left(\left\|\boldsymbol{z}-\boldsymbol{y}^{i}(j)\right\|\right)
$$




\subsection{Optimization and Error Measurement}

We have used ICA as a preprocessing step, because (3) can be written as

$$
J(\boldsymbol{W})=\sum_{j=1}^{d} \sum_{i=1}^{m} H\left(y_{i}^{j}\right)-\sum_{j=1}^{d} I\left(y_{1}^{j}, \ldots, y_{m}^{j}\right),
$$

Then, the minimization of (3) is equivalent to the maximization of the mutual information $\left(I\left(y_{1}^{j}, \ldots, y_{m}^{j}\right)\right.$ for all $\left.j\right)$ within the subspaces. To this end, 1-dimensional (1D) D Jacobi-rotations can be applied between the components. Details can be found in 1516. 1D global search is executed in each 1D optimization. An iteration cycle is made of $m^{2} d(d-1) / 2$ steps of $1 \mathrm{D}$ optimization tasks, which is much less demanding than the exhaustive search for optimal rotation in $\mathbb{R}^{m d \times m d}$. According to [10] in some cases, only 90 degree rotations (i.e., permutations of the components between subspaces) were allowed. Cycles are repeated until convergence.

Note also that if the ISA algorithm works properly, then the product of the estimated separation matrix $\boldsymbol{W}$ and the original mixing matrix $\boldsymbol{A}$ produces a permutation matrix made of $m \times m$ blocks. We measure the distance of $\boldsymbol{W} \boldsymbol{A}$ and the permutation matrix by using a generalization of the Amari-distance [22]. Let $b_{i j}$ denote the sum of the absolute values of elements at the intersection of the $i(m-1)+1, \ldots, i m$ rows and the $j(m-1)+1, \ldots, j m$ columns of matrix $\boldsymbol{W} \boldsymbol{A}$. Then the generalized Amari-distance $\rho(\boldsymbol{A}, \boldsymbol{W})$ is defined as follows:

$$
\rho(\boldsymbol{A}, \boldsymbol{W}) \doteq \frac{1}{2 d} \sum_{i=1}^{d}\left(\frac{\sum_{j=1}^{d}\left|b_{i j}\right|}{\max _{j}\left|b_{i j}\right|}-1\right)+\frac{1}{2 d} \sum_{j=1}^{d}\left(\frac{\sum_{i=1}^{d}\left|b_{i j}\right|}{\max _{i}\left|b_{i j}\right|}-1\right) \geq 0
$$

Clearly, $\rho(\boldsymbol{A}, \boldsymbol{W})=0$ iff $\boldsymbol{W} \boldsymbol{A}$ is a permutation matrix made of $m \times m$ blocks. Amari distance is a non-monotonic function of our objective, but low Amari distance is a good sign of the success of the optimization.

\section{ISA Using Innovations}

The innovation process $\hat{\boldsymbol{s}}(t)$ of a stochastic process $\boldsymbol{s}(t)$ can be written as

$$
\hat{\boldsymbol{s}}(t)=\boldsymbol{s}(t)-\boldsymbol{E}(\boldsymbol{s}(t) \mid t, \boldsymbol{s}(t-1), \boldsymbol{s}(t-2), \ldots),
$$

see e.g., 17] and the cited references. In other words, the innovation process is the error of the best prediction. Estimation of the innovation process can be performed by approximating the conditional expectation of Eq. (8), which is the best prediction of $\boldsymbol{s}(t)$ given its past in the least mean-square sense. This regression problem can be approximated by ordinary linear AR or by more sophisticated nonlinear predictions.

A special group of time-dependent stochastic processes are formed by $m$ dimensional $\tau$-order AR processes:

$$
\boldsymbol{y}(t)=\boldsymbol{F}_{1} \boldsymbol{y}(t-1)+\ldots+\boldsymbol{F}_{\tau} \boldsymbol{y}(t-\tau)+\boldsymbol{\epsilon}
$$


where $\boldsymbol{F}_{p}$ are $m \times m$ matrices and $\boldsymbol{\epsilon}$ is an $m$-dimensional i.i.d. noise. Note that for AR processes the innovation is equivalent to the $\boldsymbol{\epsilon}$ noise. If such processes are mixed in the ISA problem, then the task is to identify and 'subtract' the deterministic part of the temporal process and to recover the mixed, but i.i.d. noises. Mixed noise components then enable the ISA procedure.

In the ISA problem, the innovation process of the mixed input is the same as the mixture of the innovations of the original processes

$$
\boldsymbol{A} \hat{\boldsymbol{y}}(t)=\boldsymbol{x}(t)-\boldsymbol{E}(\boldsymbol{x}(t) \mid t, \boldsymbol{x}(t-1), \boldsymbol{x}(t-2), \ldots)=\hat{\boldsymbol{x}}(t)
$$

because of the linearity of the expectation operator. Mixing matrix $\boldsymbol{A}$ is the same for both the original and for the innovation processes. Therefore an ISA estimation which operates on the innovation process identifies the desired model.

\subsection{Algorithm}

We extended the ISA algorithm of [15]: We preprocessed the mixed data by the autoregressive model of [23]. The order of the AR model was also estimated. In the next step, traditional ICA processing was used on the gained innovation process, i.e., on the error of the prediction. This step was followed by a series of Jacobi-rotations. For all rotations the global minimum of cost function $J$ was computed, where entropy was estimated through Eq. (4) or Eq. (5). For all Jacobi-rotations, 1D global optimizations using exhaustive search for each single rotational angle $\theta \in\left[-\frac{\pi}{2}, \frac{\pi}{2}\right]$, or, only simple permutations were made.

\section{Computational Experiments}

First, we tested ISA on an artificial problem of mixed processes with known innovation processes. 2nd-order AR process were generated; the coefficient matrices of the AR process were selected randomly. Each subspace had 3 dimensions (there were 6 subspaces in total). Independent noise was added from six different 3D wireframe shapes used as sampling distributions (Fig. 1). The data, which consisted of $6 \times 3$ dimensional vectors, were mixed with random matrix $\boldsymbol{A} \in \mathbb{R}^{18 \times 18}$.

Here we ensured that an AR model is sufficient for the estimation of the innovations. Plain ISA was not able to separate the independent sources from the data (Fig. 1(e) . The combination of the autoregressive model and the ISA algorithm could, however, recover the original distributions (Figs. 1(d) and 1(f)] .

In another experiment, we used 6 different facial images with $50 \times 50$ pixels (Fig. 2(a) . The pixel values were linearly scaled and truncated to integers such that their sum was 100,000 for each image. Then we scanned the images from left-to-right and from top-to-bottom and took the $2 \mathrm{D}$ coordinate samples of the pixels as many times as directed by the value of each pixel. This is referred to as the 'pixelwise' procedure in the caption of Fig. 2. In this case, ISA could not find the proper subspaces because the sampling is very far from being temporally independent (Figs. 2(c) and 2(e). The problem is not an AR problem, there are 


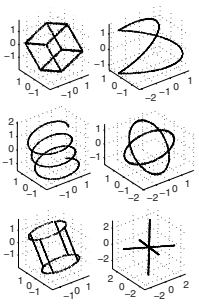

(a)

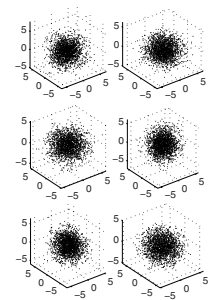

(b)

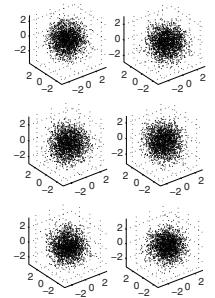

(c)
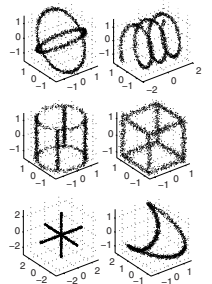

(d)

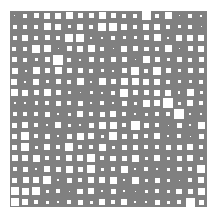

(e)

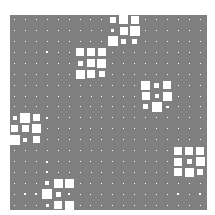

(f)

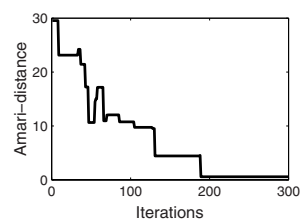

(g)

Fig. 1. ISA results for the 3D illustrative example

(a): noise of AR processes, (b): AR processes before mixing, (c): innovations, (d): estimated sources of innovations, (e): Performance without using innovations, (f): Performance with innovations, $(\mathrm{g})$ : Amari distance. Performance is shown in the form of Hinton-diagrams on the product of mixing and estimated separation matrices.

strong echoes in these 'processes' from one line to another one. Arguably, the 'processes' are deterministic. Nevertheless, when we used the innovation, ISA estimated the subspaces properly as shown in Figs. 2(d) and 2(f)] Figures were produced using Eq. (5), but both Eqs. (4) and (5) gave similar results.

\section{Discussion and Conclusions}

We have introduced the innovation process into independent subspace analysis. This step is useful if the sources have a temporal structure, but the noises in the processes are independent. A 2D problem using 'processes' generated from mixtures of facial pictures was also demixed by the innovationX process.

The concept of innovation in blind source separation techniques and the fact that it extends the range of addressable problems for ICA is not new. Autoregressive processes were used in [17]18 19]2425] for modelling the independent sources. Some ICA algorithms assume that independent sources have different autocorrelation structures and use temporal second-order correlations. If this restriction is not fulfilled, e.g., if the sources have approximately the same distributions, then separation may fail. Generalization to the multi-dimensional case is not trivial. 

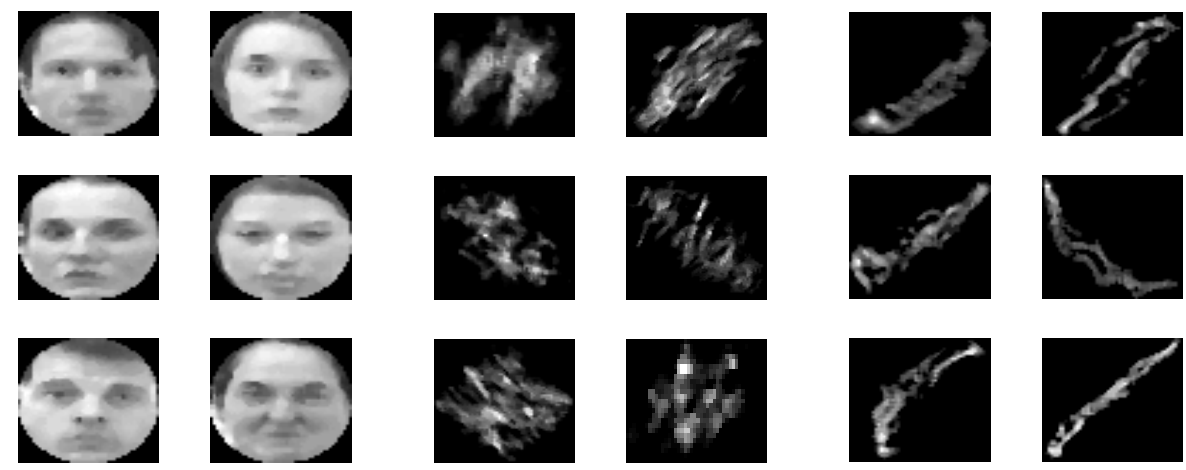

(a)

(b)

(c)
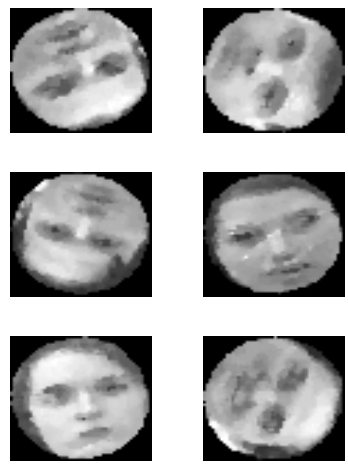

(d)

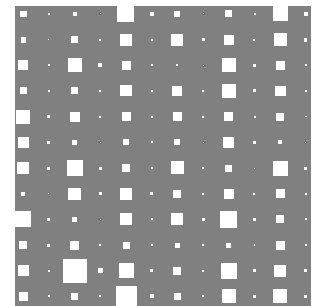

(e)

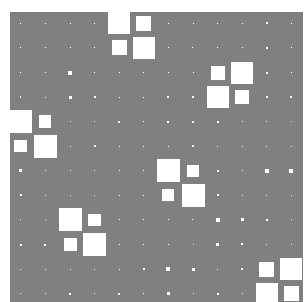

(f)

Fig. 2. Example when multi-dimensional samples drawn pixelwise

(a): original facial images, (b): mixed sources, pixelwise sampling, (c): ISA estimations, (d): estimations with ISA on innovations, (e): performance of ISA, (f): performance of ISA on innovations. Image quality is enhanced by median-filter.

Processes which are not stationary, are hard to manage with ISA, because the estimation of some quantities, e.g., the entropy and the expectations are hard. However, for AR ISA problems, the innovation is i.i.d. and our algorithm works properly. Hyvärinen [17] also argues that the innovation process has other valuable properties: Innovations are usually more independent from each other than the original processes, because the independence of the innovations does not imply that the original processes were independent - only the opposite is true. In summary, switching to the innovation process before processing the data can lead to more accurate estimations of the mixing matrix.

Our numerical simulations demonstrate that ISA alike to ICA may benefit from innovations. For AR processes, the underlying model remains unchanged 
when the original data are replaced by the innovations. We note that for the pixelwise case, the AR assumption is not valid, the problem is deterministic. Still, ISA on the 'innovations' produced good results. Thus, the robustness of the ISA makes the AR assumption the trick of demixing.

\section{References}

1. Jutten, C., Herault, J.: Blind separation of sources: An adaptive algorithm based on neuromimetic architecture. Signal Proc. 24 (1991) 1-10

2. Comon, P.: Independent component analysis, a new concept? Signal Proc. 36 (1994) 287-314

3. Bell, A.J., Sejnowski, T.J.: An information maximisation approach to blind separation and blind deconvolution. Neural Comp. 7 (1995) 1129-1159

4. Bell, A.J., Sejnowski, T.J.: The 'independent components' of natural scenes are edge filters. Vision Research 37 (1997) 3327-3338

5. Hyvärinen, A.: Sparse code shrinkage: Denoising of nongaussian data by maximum likelihood estimation. Neural Comp. 11 (1999) 1739-1768

6. Kiviluoto, K., Oja, E.: Independent component analysis for parallel financial time series. In: Proc. of ICONIP'98. Volume 2. (1998) 895-898

7. Makeig, S., Bell, A.J., Jung, T.P., Sejnowski, T.J.: Independent component analysis of electroencephalographic data. In: Proc. of NIPS. Volume 8. (1996) 145-151

8. Vigário, R., Jousmaki, V., Hamalainen, M., Hari, R., Oja, E.: Independent component analysis for identification of artifacts in magnetoencephalographic recordings. In: Proc. of NIPS. Volume 10. (1997) 229-235

9. Hyvärinen, A., Karhunen, J., Oja, E.: Independent Component Analysis. John Wiley, New York (2001)

10. Cardoso, J.: Multidimensional independent component analysis. In: Proc. of ICASSP'98, Seattle, WA. (1998) 1941

11. Hyvärinen, A., Hoyer, P.: Emergence of phase and shift invariant features by decomposition of natural images into independent feature subspaces. Neural Comp. 12 (2000) 1705-1720

12. Akaho, S., Kiuchi, Y., Umeyama, S.: MICA: Multimodal independent component analysis. In: Proc. of IJCNN. (1999) 927-932

13. Vollgraf, R., Obermayer, K.: Multi-dimensional ICA to separate correlated sources. In: Proc. of NIPS. Volume 14. (2001) 993-1000

14. Bach, F.R., Jordan, M.I.: Finding clusters in independent component analysis. In: Proc. of ICA2003. (2003) 891-896

15. Póczos, B., Lórincz, A.: Independent subspace analysis using $k$-nearest neighborhood distances. In: Proc. of ICANN, Warsaw, Poland. (2005) (accepted).

16. Póczos, B., Lőrincz, A.: Independent subspace analysis using geodesic spanning trees. In: Proc. of ICML, Bonn, Germany. (2005) (accepted).

17. Hyvärinen, A.: Independent component analysis for time-dependent stochastic processes. In: Proc. of ICANN, Skövde, Sweden. (1998) 541-546

18. Choi, S.: Acoustic source separation: Fundamental issues. In: Proc of. ICSP, Seoul, Korea. (1999) 505-510

19. Cheung, Y., Xu, L.: Dual multivariate auto-regressive modeling in state space for temporal signal separation. IEEE Trans. on Sys. Man. and Cyb. (33) 386-398

20. Yukich, J.E.: Probability Theory of Classical Euclidean Optimization Problems. Volume 1675 of Lecture Notes in Math. Springer-Verlag, Berlin (1998) 
21. Costa, J.A., Hero, A.O.: Manifold learning using k-nearest neighbor graphs. In: Proc. of ICASSP, Montreal, Canada. (2004)

22. Amari, S., Cichocki, A., Yang, H.: A new learning algorithm for blind source separation. In: Proc. of NIPS. Volume 8. (1996) 757-763

23. Schneider, T., Neumaier, A.: Algorithm 808: ARfit - A Matlab package for the estimation of parameters and eigenmodes of multivariate autoregressive models. ACM Trans. Math. Softw. 27 (2001) 58-65

24. Penny, W.D., Everson, R., Roberts, S.J.: Hidden Markov independent component analysis. In Giroliami, M., ed.: Advances in Independent Component Analysis. Springer (2000) 3-22

25. Pearlmutter, B.A., Parra, L.C.: A context-sensitive generalization of ICA. In: Proc. of ICONIP'96, Hong Kong. (1996) 151-157 